\title{
FFR-TC como herramienta útil para la detección de lesiones coronarias hemodinámicamente significativas
}

Fanny Meylin Caballeros Lam*

Elena Refoyo Salicio**

Gorka Bastarrika Alemañ*

* Servicio de Radiología. Clínica Universidad de Navarra. España

** Departamento de Cardiología y Cirugía Cardíaca. Clínica Universidad de Navarra. España

\section{Recibido:04/07/2019}

Aceptado: 29/05/2020

En línea: 31/07/2020

Citar como: Caballeros-Lam FM, Refoyo-Salicio E, Bastarrika-Alemañ G. FFR-TC como herramienta útil para la detección de lesiones coronarias hemodinámicamente significativas. Rev Ecocar Pract (RETIC). 2020 (Jul); 3 (2): 6-10. doi: 10.37615/retic.v3n2a3.

Cite this as: Caballeros-Lam FM, Refoyo-Salicio E, Bastarrika-Alemañ G. FFR-CT as a useful tool for the detection of hemodynamically significant coronary lesions. Rev Ecocar Pract (RETIC). 2020 (Jul); 3 (2): 6-10. doi: 10.37615/retic.v3n2a3.

\section{Palabras clave}

$\triangleright$ Enfermedad arterial coronaria

$\triangleright$ Coronariografía-TC

$\triangle$ FFR-TC

\section{Keywords \\ $\triangleright$ Coronary artery disease \\ $\triangleright$ Coronary computed tomography angiography $\triangleright F F R-C T$}

\section{RESUMEN}

La coronariografía por tomografía computarizada (C-TC) es un método diagnóstico no invasivo para la valoración de enfermedad arterial coronaria. A pesar de su alta sensibilidad y valor predictivo negativo, su especificidad es baja, en especial para valorar la repercusión funcional de las lesiones obstructivas. El cálculo de la reserva fraccional de flujo mediante C-TC (FFR-TC) es un método innovador que permite, en un mismo estudio, la combinación de características anatómicas y hemodinámicas. Su uso en la práctica clínica habitual puede ser decisivo para orientar el manejo terapéutico, reducir la tasa de pruebas invasivas innecesarias y los costes en salud.

ABSTRACT
Coronary computed tomography angiography is a non-invasive diagnostic method for the assessment of coro-
nary artery disease. Despite its high sensitivity and negative predictive value, its specificity is low, especially to deter-
mine the functional significance of obstructive lesions. The calculation of fractional flow reserve using computed
tomography angiography (FFR-CT) is an innovative method that allows, in the same study, the combination of
anatomic and hemodynamic characteristics. Its use in routine clinical practice can be decisive in guiding thera-
peutic management, reducing unnecessary invasive tests and health costs.

\section{Introducción}

El estudio de pacientes con angina estable o dolor torácico atípico a menudo conduce a la realización de múltiples pruebas diagnósticas. El objetivo principal de estas técnicas, tanto invasivas como no invasivas, es detectar la presencia de enfermedad arterial coronaria (EAC) y el grado de isquemia miocárdica, para estratificar el riesgo de cada paciente y así establecer un plan terapéutico adecuado(1)

Actualmente, la angiografía coronaria invasiva (ACI) se considera la técnica de referencia estándar para el diagnóstico de la EAC. Tradicionalmente, las decisiones con respecto a la revascularización se han basado en la evaluación visual del grado de estenosis coronaria, aunque la variabilidad interobservador existente se ha descrito como una limitación de la técnica ${ }^{(2)}$. Más aún, para las lesiones que no inducen isquemia el beneficio de la revascularización parece menos claro y la colocación de un stent coronario no está exenta de complicaciones. Las intervenciones percutáneas coronarias (IPC) con balón de angioplastia se han asociado a disfunción endotelial por lesión arterial. Algunos estudios también han descrito la presencia de disfunción vasomotora por daño focal en el endotelio de los segmentos adyacentes al stent, hecho que parece incrementar el riesgo de trombosis intra-stent o causar eventos cardiovasculares futuros ${ }^{(3)}$.

Múltiples estudios han demostrado que la valoración invasiva de las lesiones limitantes de flujo coronario mediante la estimación de la reserva fraccional de flujo (FFR) mejora los resultados clínicos. El estudio FAME demostró que únicamente el $35 \%$ de las estenosis visualmente moderadas fueron hemodinámicamente significativas mediante $F F R^{(4)}$. Por otra parte, tras 15 años de seguimiento de pacientes con dolor torácico estable y $F F R \geq 0,75$, el estudio DEFER objetivó que el pronóstico de las lesiones diferidas no tratadas de forma intervencionista es excelente. La IPC de tales estenosis, por tanto, parece no poseer ventajas e, incluso, puede provocar más infartos de miocardio en comparación con el tratamiento médico ${ }^{(5)}$. Como consecuencia, las guías actuales estipulan que la decisión de proceder a la revascularización coronaria debe regirse por el significado hemodinámico de la lesión.

Dados los avances tecnológicos e informáticos, hoy en día es posible realizar una evaluación no invasiva de la repercusión funcional de las lesiones coro- 
narias aplicando técnicas basadas en la dinámica de fluidos computacional para realizar una estimación de la FFR empleando las imágenes de coronariografía por tomografía computarizada (C-TC) como modelo anatómico. Esta prometedora tecnología proporciona, en una única prueba, una evaluación anatómica y funcional de las estenosis, con el objetivo de seleccionar adecuadamente a los pacientes que requieren tratamiento médico o angiografía invasiva.

\section{Evaluación invasiva de la enfermedad arterial coronaria}

La reserva fraccional de flujo (FFR) es el procedimiento de referencia estándar para guiar la revascularización coronaria percutánea, con recomendaciones clase IA de la European Society of Cardiology y clase IIA de la American Heart Association ${ }^{(6)}$.

La medición de la FFR se basa en la relación entre la presión arterial coronaria y el flujo sanguíneo. Durante un estado de hiperemia máxima, existe una relación lineal entre la presión coronaria y el flujo, ya que la resistencia periférica es mínima y, por consiguiente, constante. Para medir la FFR de una lesión coronaria se introduce un microcatéter equipado con un sensor de presión en la arteria coronaria. Después de la inducción de hiperemia máxima, usando un agente vasodilatador intravenoso o intraarterial, se registra e gradiente de presión a través de la lesión, y se calcula la FFR como la presión coronaria media distal a la estenosis dividida por la presión aórtica media. Las estenosis coronarias con una FFR $<0,75$ son hemodinámicamente significativas, mientras que las lesiones con una FFR $>0,80$ excluyen isquemia, con un valor predictivo negativo del 95\%, existiendo una zona gris entre ambos valores $^{(2)}$.

Sin embargo, a pesar de la evidencia que respalda el uso de la FFR invasiva y las recomendaciones de las guías actuales, la implementación rutinaria de la FFR en los procedimientos de revascularización sigue siendo baja (se estima que se emplea únicamente en un 10-20\% de los procedimientos realizados) (2). Según la última encuesta internacional realizada por la Asociación Europea de Intervenciones Cardiovasculares Percutáneas, más del 70\% de las decisiones sobre el manejo de lesiones moderadas, se basaron únicamente en la apariencia angiográfica(7).

Las razones que pueden explicar la infrautilización de la FFR incluyen su naturaleza invasiva, la necesidad de instrumentos costosos adicionales, la duración del procedimiento y el miedo a los riesgos relacionados con la instrumentación dentro de las arterias coronarias. Además, la idea de que los pacientes con estenosis estimadas visualmente pueden beneficiarse de la intervención aún parece estar profundamente arraigada.

\section{Evaluación no invasiva de la enfermedad arterial coronaria}

En pacientes con riesgo intermedio de EAC significativa, las recomendaciones actuales para la selección de la técnica no invasiva óptima son amplias.

Estas recomendaciones se basan en la probabilidad de tener enfermedad coronaria, en las preferencias y características de cada paciente, en la disponibilidad de las distintas técnicas y en la experiencia de cada centro ${ }^{(8)}$.

- Tomografía computarizada de emisión monofotónica (SPECT). Es una técnica útil para evaluar la perfusión miocárdica de forma cualitativa o semicuantitativa. Presenta una sensibilidad del $87 \%$ y una especi- ficidad del 70\% para la detección de EAC anatómicamente significativa respecto a $\mathrm{ACl}$ y una sensibilidad del $73 \%$ y una especificidad del $83 \%$ para la detección de EAC funcionalmente significativa respecto a la $\mathrm{FFR}^{(9)}$. Dentro de las limitaciones de esta técnica se encuentran su baja resolución espacial y el artefacto de atenuación que limita su especificidad. La SPECT dinámica con tecnecio es una nueva propuesta cuantitativa que podría mejorar la valoración de la presencia de isquemia miocárdica ${ }^{(2)}$.

- Resonancia magnética (RM) cardíaca con estrés. Ofrece la capacidad de evaluar de forma cualitativa, semicuantitativa o cuantitativa la perfusión miocárdica en reposo y estrés. Es la prueba de referencia para estudiar la función cardíaca y permite caracterizar el miocardio de manera no invasiva. Presenta una sensibilidad y especificidad del $90 \%$ y 80\% para la detección de EAC anatómicamente significativa respecto a $\mathrm{ACl}$ y una sensibilidad y especificidad del $89 \%$ y $87 \%$ para la detección de EAC funcionalmente significativa respecto a la FFR ${ }^{(9)}$. Sin embargo, su uso no está del todo extendido dada su limitada disponibilidad en algunos sitios, su elevado coste y alto consumo de tiempo en adquisición y post-procesado.

- Coronariografía por tomografía computarizada (C-TC) (Figura 1 y Figura 2). Es una prueba rápida y relativamente accesible. En la última década se ha convertido en la principal herramienta para descartar EAC, valorar la anatomía coronaria y establecer el grado de estenosis. Presenta una sensibilidad y especificidad del $97 \%$ y $78 \%$ para la detección de EAC anatómicamente significativa comparado con la $\mathrm{ACl}$. Respecto a la FFR, posee una sensibilidad del 93\% y una especificidad únicamente del 53\% para la detección de EAC funcionalmente significativa $^{(9)}$. Dentro de sus limitaciones se encuentra la sobrestimación de la severidad de las lesiones coronarias en comparación con la $\mathrm{ACl}$, en especial en placas severamente calcificadas, así como un pobre valor predictivo en la identificación de las estenosis hemodinámicamente significativas ${ }^{(2)}$.

Sin embargo, ofrece un gran detalle anatómico del árbol coronario que puede ser de interés clínico. La cuantificación de la FFR de forma no invasiva, utilizando los datos obtenidos mediante la C-TC es una de las propuestas más recientes para obtener en una única prueba de imagen información estructural y funcional de la vascularización coronaria.

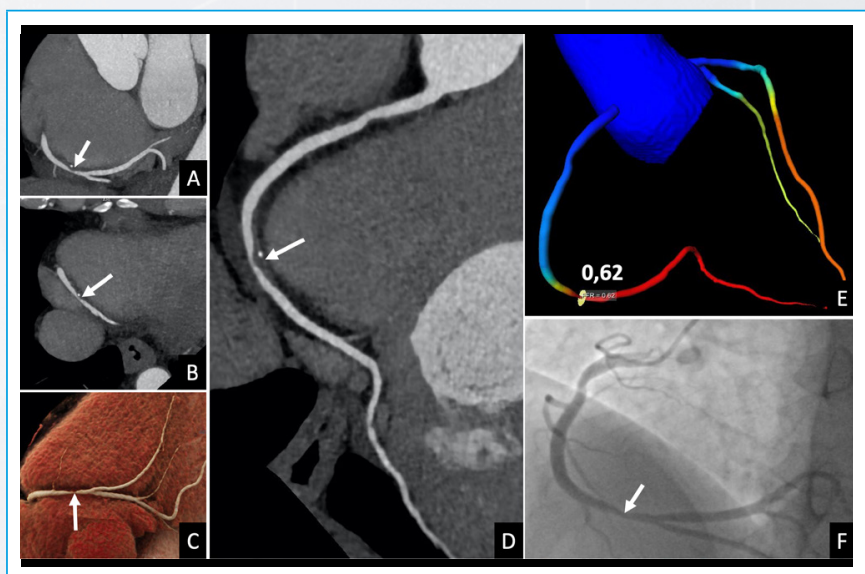

Figura 1. Coronariografía-TC en paciente con hipercolesterolemia y antecedentes familiares de cardiopatía isquémica que acudió por dolor torácico inespecífico al realizar ejercicio. El estudio demostró una placa predominantemente no calcificada en el segmento distal de la arteria coronaria derecha que provocaba estenosis $>70 \%$ (flechas en A, B, C y D). En dicha lesión se estimó una FFR-TC de 0,62 (E). Se solicitó coronariografía, que demostró la estenosis (flecha en F). El paciente fue tratado mediante implantación de stent 


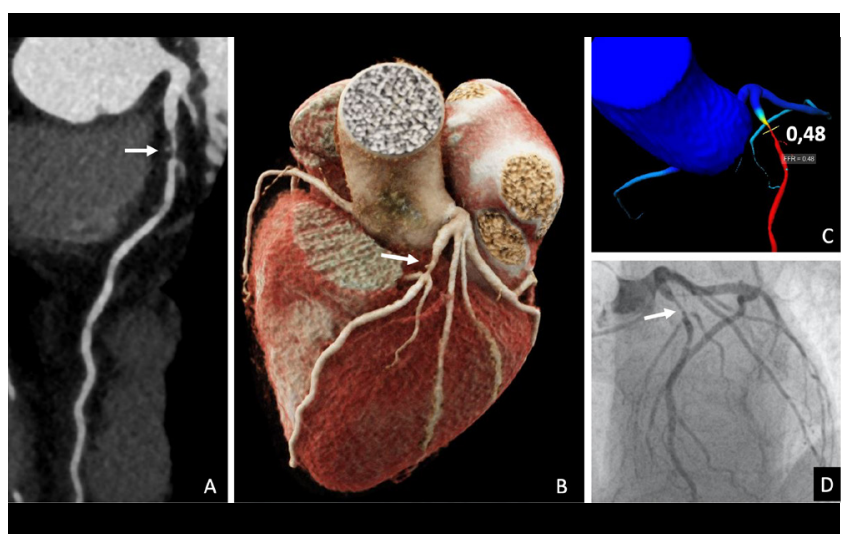

Figura 2. Coronariografía-TC en paciente que acudió por disnea de esfuerzo. El estudio demostró una placa no calcificada en el segmento proximal de la descendente anterior, que provocaba una estenosis $>70 \%$ (flechas en A y B) e implicaba al origen de la septal dominante y de la primera rama diagonal. En dicha lesión se estimó una FFR-TC de 0,48 (C). Se solicitó una coronariografía, que demostró la estenosis (flecha en D). El paciente fue tratado mediante la implantación de stent y la dilatación del ostium de la septal dominante con balón

\section{Fundamentos físicos y aspectos técnicos de la FFR-TC}

La obtención de modelos fisiológicos a partir de datos anatómicos de imagen tras aplicar principios informáticos de dinámica de fluidos computacional se basa en los siguientes tres principios(6):

- El flujo sanguíneo coronario total se puede calcular en relación con la masa miocárdica. Esto se debe a que el flujo sanguíneo coronario es proporcional a la demanda de oxígeno del miocardio en reposo.

- La resistencia de la microvasculatura en reposo es inversamente proporcional a la luz del vaso. Las arterias, tanto normales como patológicas, adaptan su tamaño en función de la cantidad de flujo.

- La microcirculación coronaria tiene una respuesta vasodilatadora predecible a la adenosina. La administración exógena de adenosina provoca un estado de hiperemia máxima, por una relajación de las células musculares lisas que recubren las arteriolas de resistencia, con un consecuente aumento del flujo sanguíneo coronario. En casos fisiológicos, cuando el miocardio carece de oxígeno, el ATP provoca una liberación de adenosina endógena. Esta respuesta predecible permite la simulación de la hiperemia máxima en el modelo computacional.

Los avances en la dinámica de fluidos computacional permiten la determinación de la FFR a partir de imágenes estáticas obtenidas mediante C-TC. La C-TC se realiza utilizando protocolos de adquisición estándar, sin necesidad de adquirir imágenes adicionales o mayor exposición a radiación y no es necesario administrar adenosina.

\section{Adquisición de la FFR-TC}

La elaboración de la FFR-TC requiere tres pasos principales ${ }^{(10)}$ :

1. Elaboración de un modelo anatómico y geométrico de la raíz aórtica y de las arterias coronarias, específico para cada paciente. Para ello se realiza una segmentación semiautomática del contorno y lumen de las arterias coronarias principales y sus ramas, así como de la masa del ventrículo izquierdo.
2. Utilización de un modelo matemático de la fisiología coronaria durante máxima hiperemia, mediante leyes de ajustes alométricos, para establecer las condiciones que representa gasto cardíaco, presión aórtica y resistencia de la microcirculación.

3. Aplicación de una ecuación computacional de las leyes físicas de la dinámica de fluidos, mediante la modelización de la sangre como fluido newtoniano y la modelización de la turbulencia utilizando ecuaciones de Navier-Stokes.

\section{Validación clínica}

Para evaluar la precisión diagnóstica de la FFR-TC se han realizado múltiples estudios multicéntricos prospectivos, destacando los siguientes:

- Estudio DISCOVER-FLOW. Fue el primero en utilizar la FFR-TC, incluyendo a 103 pacientes con EAC estable, a quienes se les realizó C-TC, ACl, FFR invasiva y FFR-TC. La FFR-TC aumentó la especificidad de estenosis hemodinámicamente significativas a un $82 \%$ comparado con el $40 \%$ de especificidad de C-TC. Además, demostró una mejora del $42 \%$ en la precisión diagnóstica (84\% para FFR-TC frente a 59\% para C-TC), destacando un alto rendimiento diagnóstico para la detección de lesiones coronarias causantes de isque$\operatorname{mia}^{(6)}$.

- Estudio NXT. Incluyó a 251 pacientes con EAC estable, programados para IPC. Se prestó especial atención en obtener imágenes de TC de alta calidad, administrando nitroglicerina y betabloqueantes, así como ajustando los protocolos de imagen al tamaño corporal del paciente para minimizar el ruido. Demostraron una mejora sustancial en la precisión diagnóstica por paciente y por lesión para la FFR-TC en comparación con la C-TC (81\% frente a $53 \%$ y $86 \%$ frente a $65 \%$, respectivamente), lo que resultó en una mayor especificidad, con sensibilidad comparable ${ }^{(11)}$

- Estudio PLATFORM. Incluyo a 584 pacientes con EAC recién diagnosticada. Como objetivo buscó evaluar cómo la FFR-TC afectaba la necesidad de ACl. Asignaron a los pacientes en dos grupos: directamente a IPC o a C-TC/FFR-TC, con la posible cancelación de la IPC planificada basada en los resultados. La estrategia C-TC/FFR-TC resultó en la cancelación del $61 \%$ de IPC planeadas previamente, y ninguno de estos pacientes presentó eventos cardiovasculares tras 1 año de seguimiento. La estrategia C-TC/FFR-TC como es lógico demostró ser más costeefectiva, aunque como ya se ha dicho se cancelaron un $61 \%$ de los IPC programados (11).

- Estudio ADVANCE. Incluyó a 5.083 pacientes con EAC estable, en los que se evaluaron los resultados clínicos y recursos utilizados, tras el tratamiento escogido según la FFR-TC. La utilización de la FFR-TC reclasificó el manejo del $66,9 \%$ de los pacientes. El $72,3 \%$ de los pacientes sometidos a ACI con FFR-TC $<0,80$ fueron revascularizados. Únicamente un 14,4\% tuvieron enfermedad no obstructiva. No ocurrió ninguna muerte/infarto de miocardio durante el seguimiento a los 90 días en pacientes con FFR-TC $>0,80$, mientras que ocurrió un $0,6 \%$ de MACE y 0,3\% de muerte/infarto de miocardio en sujetos con FFR-TC $<0,80^{(12)}$. La continuación del estudio ADVANCE, tras 1 año de seguimiento, demostró tasas bajas de eventos adversos, menor tasa de revascularización y menor incidencia de MACE, muerte cardiovascular o infarto de miocardio en pacientes con FFR-TC $>0,80$ en comparación con pacientes con FFR-TC $<0,80(0,2 \%$ frente a $0,8 \% ; p=0,01)^{(13)}$.

- Estudio SYNTAX. Incluyó a 77 pacientes con EAC estable multivaso, con el objetivo de valorar la viabilidad y validación del score funcional SYNTAX derivado de FFR-TC. Dio resultados similares a los obtenidos de forma invasiva, reclasificando al 30\% de los pacientes del tercil de riesgo alto-intermedio al tercil de riesgo bajo. Además, demostró que la FFR-TC tiene buena precisión en la detección de lesiones funcionalmente significativas en pacientes con enfermedad multivaso ${ }^{(11)}$. 


\section{Limitaciones}

Uno de los factores que influye significativamente en la cuantificación de la FFR-TC es la calidad de las imágenes obtenidas mediante C-TC. Los artefactos de movimiento, el endurecimiento del haz por la presencia de ateromatosis calcificada coronaria y el ruido de la imagen desempeñan un papel importante. Estos problemas pueden minimizarse mediante el uso de betabloqueantes para reducir la frecuencia cardíaca y la variabilidad de la frecuencia cardíaca y mediante la administración de nitratos sublinguales para dilatar las arterias coronarias. Dado que la exactitud de la FFR-TC depende de la precisión con la que se lleve a cabo la segmentación coronaria, la calidad de la imagen debe ser un objetivo primordial en la adquisición de la C-TC(6).

Por otra parte, para el cálculo computacional de la FFR-TC se toman en cuenta determinadas premisas fisiológicas que no tienen por qué cumplirse siempre, de manera que la precisión diagnóstica de la FFR-TC en comparación con la FFR puede verse afectada por las diferencias específicas de cada paciente. Por ejemplo, la respuesta de la microcirculación a los vasodilatadores y las condiciones fisiológicas individuales pueden afectar a parámetros como la densidad de fluidos y viscosidad. La viscosidad es asumida a partir de la concentración de hematocrito/hemoglobina y, cuando está en el rango normal, tiene una influencia mínima en la FFR-TC. Sin embargo, bajo condiciones de anemia severa, la viscosidad reducida puede afectar el cálculo de la FFR-TC. La magnitud de tal impacto aún no se conoce.

La interpretación de la FFR-TC requiere una apreciación de los valores más distales al área de estenosis, y no sólo inmediatamente a nivel de la misma, como suele hacerse en la FFR invasiva. Aún no existe una distancia específica estandarizada, desde el área de estenosis, para realizar dicha medición. En un pequeño estudio de cohortes, Kueh et al. demostraron que el $44 \%$ de los pacientes con FFR-TC $<0,8$ en los vasos distales tenían una FFR-TC $>0,8$ a menos de $2 \mathrm{~cm}$ de la estenosis angiográfica ${ }^{(14)}$. Es posible que se requiera evaluar con mayor precisión una aproximación estandarizada de la lesión para la valoración de la FFR-TC.

Hasta la fecha no se ha valorado la FFR-TC en pacientes con bypass coronario o sospecha de reestenosis en el stent.

Además de las limitaciones técnicas, existen otros obstáculos generales que hasta ahora han impedido una integración más generalizada de la FFR-TC en los flujos de trabajo rutinarios. Por ejemplo, el tiempo de posprocesado para la determinación la FFR-TC puede ser largo y es variable según el método de evaluación utilizado. El algoritmo actualmente disponible comercialmente, aprobado por la FDA, requiere que los datos de la C-TC sean enviados a un laboratorio central para su análisis, donde los resultados de la FFR-TC son devueltos en 1-4 horas. Esto puede limitar la aplicación clínica especialmente en situaciones de emergencia ${ }^{(2)}$.

Existen otros algoritmos que se pueden utilizar localmente en estaciones de trabajo y que permiten determinar la FFR-TC en aproximadamente 45 minutos o menos, pero requieren la interacción del médico y, hasta la fecha, muchos son prototipos en investigación que aún no han sido aprobados por la FDA. En el futuro, la utilización del deep-learning en el análisis FFR-TC tiene el potencial de reducir los tiempos de cálculo.

\section{Conclusión}

La FFR-TC se ha validado como una prueba no invasiva para la evaluación anatómica y funcional de la severidad de la EAC, demostrando alta precisión diagnóstica en comparación con la FFR invasiva.
La evidencia actual muestra que la FFR-TC puede ser un complemento importante de la C-TC para la toma de decisiones clínicas y el manejo del paciente, reduciendo la tasa de pruebas invasivas innecesarias y el coste en atención sanitaria.

\section{Ideas para recordar}

- El beneficio clínico de la revascularización de lesiones coronarias se limita a aquellas lesiones causantes de isquemia y no únicamente al grado de estenosis.

- La FFR-TC se puede estimar de forma no invasiva, mediante métodos de dinámica de fluidos computacional aplicados a protocolos estándar de C-TC, sin necesidad de mayor exposición a radiación ni administración de adenosina.

- Múltiples estudios han demostrado un aumento en la precisión diagnóstica y especificidad de la C-TC estándar al añadir el cálculo de FFR-TC.

- La FFR-TC, aunque todavía posee limitaciones para su uso rutinario, se perfila como una técnica de gran utilidad clínica para establecer el significado funcional de la estenosis coronaria.

\section{Bibliografía}

1. Chinnaiyan KM, Akasaka T, Amano T, et al. Rationale, design and goals of the Heart Flow assessing diagnostic value of non-invasive FFRCT in Coronary Care (ADVANCE) registry. J Cardiovasc Comput Tomogr 2017; 11 (1): 62-67.

2. Tesche C, De Cecco CN, Albrecht MH, et al. Coronary CT angiography-derived fractional flow reserve. Radiology 2017; 285 (1): 17-33.

3. Lim SH, Flammer AJ, Yoon MH, et al. The long-term effect of coronary stent on epicardial and microvascular endothelial function. Circ Cardiovasc Interv 2012; 5 (4): 523-529.

4. Mathew RC, Gottbrecht M, Salerno M. Computed tomography fractional flow reserve to guide coronary angiography and intervention. Intervent $\mathrm{Car}$ diol Clin 2018; 7 (3): 345-354.

5. Zimmermann FM, Ferrara A, Johnson NP, et al. Deferral vs. performance of percutaneous coronary intervention of functionally non-significant coronary stenosis: 15-year follow-up of the DEFER trial. Eur Heart J 2015; 36 (45): 3182-3188.

6. Zarins CK, Taylor CA, Min JK. Computed fractional flow reserve (FFTCT) derived from coronary CT angiography. J Cardiovasc Trans/ Res 2013; 6 (5): 708-714.

7. Toth GG, Toth $B$, Johnson NP, et al. Revascularization decisions in patients with stable angina and intermediate lesions: results of the international survey on interventional strategy. Circ Cardiovasc Interv 2014; 7 (6): 751759.

8. Knuuti J, Wijns W, Saraste A, et al. 2019 ESC Guidelines for the diagnosis and management of chronic coronary syndromes. Eur Heart J 2020; 41 (3): 407477.

9. Knuuti J, Ballo H, Juarez-Orozco LE, et al. The performance of non-invasive tests to rule-in and rule-out significant coronary artery stenosis in patients with stable angina: a meta-analysis focused on post-test disease probability. Eur Heart J 2018; 39 (35): 3322-3330

10. Taylor CA, Fonte TA, Min JK. Computational fluid dynamics applied to cardiac computed tomography for noninvasive quantification of fractional flow reserve: scientific basis. J Am Coll Cardiol 2013; 61 (22): 2233-2241.

11. Ball C, Pontone G, Rabbat M. Fractional flow reserve derived from coronary computed tomography angiography datasets: the next frontier in noninvasive assessment of coronary artery disease. Biomed Res Int 2018; 2018: 2680430.

12. Fairbairn TA, Nieman $K$, Akasaka T, et al. Real-world clinical utility and impact on clinical decision-making of coronary computed tomography angiogra- 
phy-derived fractional flow reserve: lessons from the ADVANCE Registry. Eur Heart J 2018; 39 (41): 3701-3711.

13. Patel MR, Nørgaard BL, Fairbairn TA, et al. 1-Year impact on medical practice and clinical outcomes of FFRCT The ADVANCE Registry. JACC CardiovasC Imaging 2020; 13 (1 Pt 1): 97-105.
14. Kueh SH, Mooney J, Ohanaet M, et al. Fractional flow reserve derived from coronary computed tomography angiography reclassification rate using value distal to lesion compared to lowest value. J Cardiovasc Comput Tomogr 2017; 11 (6): 462-467 